\title{
Las psicosis de la epilepsia: presentación de un caso clínico y revisión de la literatura.
}

The psychosis of epilepsy: a case report and literature review.

\author{
Fernando Lázaro Perlado ${ }^{\text {a }}$, Manuel Conde Rivas ${ }^{\text {b }}$, María Victoria Caminero Olea ${ }^{\text {c }}$, Jon Baraia- \\ zarra Ruiz ${ }^{\text {d. }}$
}

\begin{abstract}
${ }^{a}$ Médico Adjunto; Osakidetza- Servicio Vasco de Salud; Red de Salud de Mental de Bizkaia; Hospital de Bermeo; Bermeo, Bizkaia, España. ${ }^{b}$ Médico Adjunto; Osakidetza-Servicio Vasco de Salud; Red de Salud de Mental de Bizkaia; Hospital de Bermeo; Bermeo, Bizkaia, España. ${ }^{c}$ Médico especialista en Medicina Familiar y Comunitaria; Osakidetza-Servicio Vasco de Salud; Comarca Uribe-Kosta; Centro de Salud de

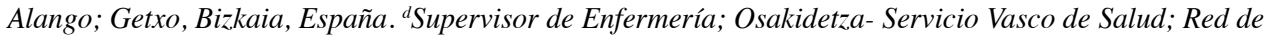
Salud de Mental de Bizkaia; Hospital de Bermeo; Bermeo, Bizkaia, España.
\end{abstract}

Correspondencia: Fernando Lázaro Perlado (fernando.lazaroperlado@osakidetza.net)

Recibido: 13/08/2012; aceptado con modificaciones: 17/12/2012

\begin{abstract}
RESUMEN: Presentamos el caso de un varón de 72 años con un diagnóstico previo de epilepsia del lóbulo temporal y esquizofrenia paranoide, que mostraba episodios de psicosis con sintomatología pleomórfica y atípica que no respondía bien al tratamiento antipsicótico. El paciente no presentaba un deterioro en su personalidad y había demostrado un buen ajuste y funcionamiento social. Tras revisar el caso se llega a cuestionar el diagnóstico previo de esquizofrenia paranoide proponiendo el diagnóstico alternativo de epilepsia del lóbulo temporal: crisis comiciales con sintomatología psicótica. Se instaura el tratamiento adecuado con éxito lo que permite el alta del paciente a su domicilio. Revisamos la literatura en la materia, concluyendo que las psicosis de la epilepsia han de tenerse en cuenta en casos de psicosis atípicas, resistentes a tratamiento en personas con buen funcionamiento psico-social sin deterioro en su personalidad. PALABRAS CLAVE: epilepsia del lóbulo temporal; psicosis comiciales; psicosis de la epilepsia; psicosis de la epilepsia tipo esquizofrenia.

CONFLICTO DE INTERESES: Ninguno.
\end{abstract}

ABSTRACT: We describe the case of a 72 year old man with a diagnosis of temporal lobe epilepsy and paranoid schizophrenia, who presented atypical psychotic episodes which did not respond to anti-psychotic medication. His personality had not deteriorated and he showed good social adjustment and functioning. After reviewing the case, we challenged the diagnosis of paranoid schizophrenia, proposing an alternative diagnosis of temporal lobe epilepsy with ictal psychosis. He was successfully treated and discharged home. We review the literature on the subject. The psychosis of epilepsy are a diagnostic option which must be seriously considered in cases of treatment resistant atypical psychosis in subjects with good pre-morbid functioning and social adjustment free from personality decline.

KEY WORDS: temporal lobe epilepsy; ictal psychosis; psychosis of epilepsy; schizophrenia like psychosis of epilepsy.

DECLARATION OF INTEREST: None. 
Presentamos el caso de un varón de 72 años que ingresa voluntariamente en nuestra unidad de rehabilitación en agosto del 2010 con un "cuadro confusional secundario a crisis comiciales (tonico-clónicas y ausencias) de repetición, con alteraciones conductuales que impiden el tratamiento ambulatorio". Previamente había sido diagnosticado de esquizofrenia paranoide y epilepsia del lóbulo temporal.

Ingresó en la unidad de agudos por presentar alteraciones de la conducta en un marco confusional. Durante el mismo presentó varias crisis comiciales y de ausencia que fueron controladas ajustando su tratamiento anti-convulsivo. Sin embargo, persistieron las ideas delirantes de contenido místico-religioso y algún componente paranoide en relación a su tratamiento y estancia en el hospital. Desde allí se le traslada a nuestro centro.

En el momento de ingreso el tratamiento farmacológico pautado era: carbamacepina (1000 mg/día), valproato (1300 mg/día), pimozida (8 mg/día) y lorazepam (1-2 mg si insomnio).

Fue diagnosticado de epilepsia del lóbulo temporal a los 17 años, debido a una lesión cerebral cuyo origen ha sido imposible de esclarecer. Tuvo su primer ingreso psiquiátrico en nuestro hospital cuando el paciente tenía 34 años de edad. El cuadro clínico que presentaba en ese momento eran crisis comiciales desencadenas por la suspensión del tratamiento previa a la realización de un EEG de control. Tras las crisis presentó un cuadro delirante de contenido místico - religioso y alteraciones de la conducta. La familia informó en ese momento que había presentado un episodio similar 7 años antes, coincidiendo también con la aparición de crisis comiciales, y que remitió con un ajuste farmacológico para su patología comicial.

En los años posteriores tuvo múltiples ingresos (aproximadamente 15) en diferentes centros hospitalarios. El motivo de estos ingresos era siempre similar. Cuadros delirantes de contenido místico religioso (Dios le había hablado y le había dicho que él iba a ser Papa, el mundo iba a acabar pronto, la Iglesia no sigue el camino de Cristo...) y alteraciones de conducta: episodios de agitación con amenazas verbales y físicas, negarse a comer y a beber. El paciente acaba siendo diagnosticado de esquizofrenia paranoide.

Durante los últimos 3 años hacía seguimiento en su CSM de referencia sin mayores problemas, con excepción de abandono de la medicación durante los brotes psicóticos.

No hay ningún dato de interés en su desarrollo psicomotor, infancia o adolescencia previo al inicio de si epilepsia. Completó estudios de delineante, y trabajó en esa función con buen rendimiento hasta que fue jubilado por enfermedad a los 52 años.

Se casó con 30 años. La relación con su mujer era buena pero enviudó a los 47 años. Tuvieron un hijo varón, con el que convive en la actualidad No se le conocen hábitos de consumo de tóxicos, alcohol o tabaco. Tiene dos hermanos mayores. 
No se detectaron antecedentes familiares de interés.

La valoración del estado mental en ingreso reveló a un varón de edad avanzada, bien presentado y arreglado, que mostraba una marcha "en segador" leve pero observable. Eutímico con afecto apropiado. Cooperante. Su discurso, aunque espontáneo era disártrico, sobreincluyente y tangencial. Se observó que era muy metódico y ordenado (a la hora de arreglar sus cosas, doblar la ropa...). Nos dijo que se encontraba "bien" y no se objetivaron síntomas o signos que apuntaran a un proceso afectivo. No había ideación, planificación ni intención autolítica. No presentó alucinaciones y, en ese momento no se objetivaron ideas delirantes. Nos dijo que aparte de sus problemas físicos (antecedente de hernia abdominal), no tenía problema alguno. Estaba desorientado en tiempo y lugar. No tenía conciencia de enfermedad.

En la exploración física tenía cicatrices por cirugía abdominal, hipoacusia leve, dificultad para completar el movimiento "dedo-nariz" en EESS izquierda, quadrantanopsia homonónima inferior derecha, reflejo plantar ausente en EEII izquierda, y leve "marcha en segador" con inestabilidad en la marcha.

El paciente ya había sido explorado con TAC craneal (Junio 2009) que detecta patología isquémica residual en hemisferio cerebeloso derecho y lóbulo temporal izquierdo, con moderada atrofia cortico-subcortical. No se observan cambios patológicos tras la introducción de contraste.

En vista de la sospecha diagnóstica se realiza un EEG convencional (Septiembre 2010), que se informa como: "EEG de vigilia y fase I de sueño fisiológico en el que se observa una discreta lentificación cerebral difusa".

Durante su estancia se procedió a un examen básico de su estado cognitivo usando el Mini-Mental (MMSE) y el Adenbrooke's Cognitive Examination, versión en castellano (ACEve) (1), obteniendo una mejoría mínima la segunda ocasión. Mostrándose perseverativo, concreto e incapaz de completar el test de Luria o una secuencia gestual alternativa, con marcados déficits mnésicos tanto en memoria semántica como en la capacidad de aprendizaje de nueva información, déficits en fluidez verbal, mostrando dislexia de superficie y dispraxia construccional. Los resultados del MMSE fueron de 14/30 en las dos ocasiones; puntuando en el ACEve 34/100 en agosto del 2010) y 44/100 en febrero del 2011. Estas puntuaciones denotan un deterioro cognitivo importante, posiblemente de etiología mixta, pero con un claro componente vascular.

Durante su estancia en nuestra unidad, se observaron inicialmente episodios de agitación, con amenazas verbales y físicas, y presencia de ideas delirantes de contenido místico-religioso. Estos episodios se presentaban, con mayor frecuencia al anochecer, desaparecían rápidamente tras la administración de dosis bajas de lorazepam oral.

Su tratamiento se fue ajustando. Se añadió clobazam (dosis inicial de $10 \mathrm{mg} /$ día, que se fue aumentando según tolerancia hasta un máximo de 15) a su pauta 
medicamentosa, reduciendo el pimozide hasta suspenderlo. El paciente respondió muy favorablemente. Los episodios de agitación con ideas delirantes desaparecieron por completo.

En ningún momento durante la estancia en nuestra unidad se observaron crisis comiciales con manifestaciones motoras o de ausencia.

En vista de su evolución favorable, se empezó a planificar su alta. Para ello, se acordó con su hijo un plan de alta gradual, con varios permisos a casa. Se procedió a su alta definitiva al domicilio familiar en marzo del 2010.

El diagnóstico al alta fue:

Epilepsia del Lóbulo Temporal: Crisis comiciales con sintomatología psiquiátrica (versus Esquizofrenia paranoide y Epilepsia del lóbulo temporal).

Deterioro cognitivo (demencia) de probable etiología mixta.

Su tratamiento al alta, incluía carbamacepina (1000 mg/día), valproato (1300 $\mathrm{mg} /$ día), clobazam (15 mg/día) y lorazepam (1 mg si insomnio).

\section{Las psicosis de la epilepsia}

La epilepsia es una entidad nosológica crónica y recurrente que se caracteriza por la aparición de crisis comiciales (2).

Las psicosis comiciales, o de la epilepsia, consisten en episodios psicóticos con sintomatología pleomórfica, de duración variable -desde horas hasta años- que pueden ser la única manifestación clínica de un episodio epiléptico, o consecuencia de una actividad eléctrica aberrante, mantenida o intermitente, en determinadas áreas cerebrales (3).

Se clasifican según su relación temporal con las convulsiones epilépticas o racimos ("clusters") de convulsiones (3). Así, se dividen en pericomiciales (precomiciales, comiciales y postcomiciales) e intercomiciales, éste último tipo también conocido como las Psicosis de la Epilepsia Tipo Esquizofrenia (PETE) (del inglés Schizophrenia Like Psychosis of Epilepsy, SLPE), por su semejanza clínica a la esquizofrenia (4-7).

En ocasiones, la aparición del cuadro psicótico, precede a la aparición de la epilepsia, hasta en un 17\% de pacientes con epilepsia y psicosis en algunos estudios (8).

Se acepta que las psicosis de la epilepsia son más frecuentes en sujetos con antecedentes familiares de enfermedad psicótica (9), epilepsia del lóbulo temporal y esclerosis temporal medial, sin observarse diferencias en cuanto a subtipos o patrones sintomatológicos (10).

De hecho, estudios de neuroimagen estructural han detectado diversas alteraciones en estructuras del lóbulo temporal medial, como displasia medial temporal, 
con preservación del hipocampo y agrandamiento amigdalina $(11,12)$, con estudios posteriores detectando atrofia y no agrandamiento amígdalino en sujetos con epilepsia del lóbulo temporal (13).

Como veremos más adelante, no siempre se pueden detectar anomalías en el electroencefalograma (EEG) que nos orienten hacia éste diagnóstico. Es por eso, que se deberá tener en cuenta una posible etiología epiléptica en pacientes que presentan un cuadro psicótico atípico, resistente a tratamiento o con empeoramiento sintomático a la medicación antipsicótica, que presenten factores de riesgo para la epilepsia, aunque no tengan un diagnóstico previo ni se hayan documentado crisis convulsivas (6).

Psicosis comiciales: "status epilepticus” parcial complejo.

El término "status epilepticus" parcial complejo fue acuñado por Gastaut (14) en 1956, como resultado de la observación de sintomatología productiva psicótica tras episodios de actividad epiléptica prolongada. Observamos entonces episodios psicóticos que son la manifestación clínica de una crisis epiléptica, en ausencia de convulsiones patentes.

La sintomatología del episodio psicótico es pleomórfica, con patente confusión e inaccesibilidad a la interacción con el entrevistador. Ésta incluye delirios, ilusiones y alucinaciones visuales y auditivas (en ocasiones de contenido religioso), ideas autoreferenciales, paranoia y desorganización del pensamiento. La duración de estos episodios es normalmente de horas, pero pueden durar meses o incluso años, y la sintomatología fluctúa y cicla. El cuadro clínico característico es uno de varias horas de angustia con agitación psicomotriz, delirios y alucinaciones (3).

Las psicosis comiciales, son un fenómeno poco detectado y estudiado. Muchos casos escapan un diagnóstico acertado, en parte, porque no siempre se pueden detectar en un EEG convencional. En las raras ocasiones en que se pueden detectar anomalías electroencefalográficas, éstas aparecen en el septum y, con menor intensidad, en las estructuras del lóbulo temporal medial, amígdala e hipocampo, mientras que en pacientes con esquizofrenia que también presentan epilepsia, se observa el patrón reverso: clara actividad de puntas y ondas en la región temporal medial y menor actividad epiléptica en el septum. Por estas razones, se postula que las psicosis comiciales pueden estar asociadas a actividad epiléptica en áreas límbicas profundas, cuya actividad eléctrica no se puede detectar en un EEG convencional. Así, se pueden observar anomalías conductuales sin cambios electroencefalográficos observables. Otros métodos diagnósticos como la RMN funcional quizás inicialmente validada por EEG intracraneal, sean necesarios para una valorar la prevalencia verdadera de las psicosis comiciales $(3,6,15)$. 
Otro factor que dificulta un diagnóstico acertado de manera rápida, al dificultar relacionar sintomatología productiva con alteraciones en el EEG es la normalización del registro EEG coincidente con la aparición de síntomas psicóticos, proceso conocido como "normalización forzada" (16). Es decir, mientras no se objetivan alteraciones en el estado mental del sujeto, se pueden detectar alteraciones en el EEG, las cuales desaparecen al aparecer la sintomatología psicótica. Se postula que un proceso inhibitorio continuo resulta en el control y cese de las convulsiones, con normalización en el EEG, y la consiguiente prolongación del episodio psicótico (17).

Su tratamiento consiste en controlar la actividad eléctrica aberrante, la convulsión, con fármacos antiepilépticos. En aquellos casos en los que se sospeche un "status epilepticus" de origen límbico, como causante del episodio psicótico, se aconseja tratamiento con benzodiacepinas, con ayuda de medicación antipsicótica si el cuadro es severo. Clobazam, suele ser el medicamento de elección en estos casos, en administración oral durante varios días en dosis de $20-30 \mathrm{mg} /$ día. El terapeuta tener en cuenta los efectos de los neurolépticos sobre el umbral de convulsión (3).

\section{Psicosis postcomiciales:}

Concepto desarrollado por Jackson (18) en 1875, en su trabajo "Trastornos mentales transitorios después de paroxismos epilépticos", donde se describe por primera vez, sintomatología psiquiátrica tras crisis epilépticas. En la actualidad, se acepta la definición de Kanemoto (19) que incluye cualquier episodio psicótico que aparece en un período no superior a 7 días después de la última convulsión tónico-clónica o racimo de convulsiones parciales complejas. Arbitrariamente se ha fijado la duración del episodio en no más de 2 meses (17) aunque en su trabajo seminal, Logsdail y Toone (20) demostraron episodios con una duración de hasta 3 meses.

$\mathrm{Su}$ incidencia se estima entre el $6.4 \%$ y $6.7 \%$ de la población epiléptica, siendo en algunos estudios, más frecuente que las psicosis intercomiciales (21).

Los factores de riesgo asociados con la psicosis postcomicial son: foco bitemporal (22), foco ambiguo o extratemporal, antecedentes familiares neuropsiquiátricos, EEG intercomicial anormal, y antecedentes de encefalitis. También se ha detectado una asociación con antecedentes familiares de epilepsia y trastorno psiquiátricos, que a su vez se asociaban entre ellos $(9,21,23)$.

Chang Y-T et al, observaron una asociación bidireccional entre epilepsia y trastornos psicóticos sin encontrar clara causalidad, aunque su estudio no clarifica si establecieron control para individuos con psicosis comiciales (23).

Las manifestaciones clínicas que caracterizan éstos casos incluyen (24): 
NOTAS CLÍNICAS

- Una latencia, no mayor de 7 días, entre la convulsión y la aparición de sintomatología psiquiátrica.

- La relativa corta duración del episodio.

- Sintomatología de predominio afectivo.

- La agrupación de los síntomas en ideas delirantes y psicosis afectivas.

- Aumento de la frecuencia de convulsiones tónico-clónicas secundariamente generalizadas antes de la aparición del episodio psicótico.

- La aparición de la psicosis postcomicial tras más de 10 años de epilepsia.

- Rápida respuesta a dosis bajas de antipsicóticos ó benzodiacepinas.

Los pacientes que presentan ésta patología tienden a presentar delirios de contenido religioso, o de grandeza, con un humor elevado y sentimiento de fusión mística de su cuerpo con el universo, sintomatología que según el autor, ayuda a diferenciar estos episodios de los comiciales y de los intercomiciales. También se han descrito episodios de estupor aquinético, miedo, síndrome de Capgras, o la sensación de que alguien se encuentra próximo a uno mismo todo el tiempo (19).

Se considera que éstos pacientes presentan un riesgo de suicidio alto así como de progresión a psicosis inter-comiciales o PETE (6).

No siempre se pueden documentar anomalías en el EEG que ayuden en el diagnóstico, al ser los hallazgos electroencefalográficos en los episodios psicóticos post-comiciales inconsistentes, o no detectan anormalidad alguna. De hecho, en un estudio que incluía a 53 sujetos con un diagnóstico probado de epilepsia y psicosis post-comiciales, 6 de ellos no mostraron anormalidades en videotelemetría a pesar de haber presentado convulsiones probadas 24 horas pre-ingreso (21).

Al igual que en las psicosis comiciales, el clínico debe tener en cuenta la "normalización forzada" (16) al cotejar los hallazgos en el EEG con la presentación clínica del paciente y llegar a una conclusión diagnóstica adecuada.

Para su tratamiento, se recomienda la terapia con benzodiacepinas, en ocasiones ayudados por fármacos antipsicóticos. Las psicosis postcomiciales de la epilepsia también responden a la terapia electroconvulsiva, aunque es una opción terapéutica en desuso en la actualidad $(3,25)$.

Psicosis intercomiciales o psicosis de la epilepsia tipo esquizofrenia (PETE).

Diagnosticar éste grupo de psicosis no es tarea fácil, puesto que, para poder llegar a éste diagnóstico, hay que descartar los diagnósticos de psicosis comiciales y postcomiciales (de por sí tarea complicada), tener en cuenta que los episodios de psicosis intercomiciales en los que se alternan las crisis epilépticas con los episo- 
dios psicóticos pueden ser expresión, aunque infrecuente, de una normalización forzada, además de los efectos secundarios proconvulsivos de la medicación psicotrópica (26).

Este grupo se define como las psicosis que aparecen después del comienzo de la epilepsia; pueden no estar precedidas por convulsiones patentes y aparecer en un período libre de convulsiones o entre convulsiones de repetición; los episodios duran 24 horas o más con preservación de la consciencia. Incluyen episodios tanto largos, con una duración de 1 mes o mayor, como breves, con una duración menor a un mes (5).

Éste grupo de psicosis aparece entre el $4 \%$ y el $10 \%$ de pacientes con epilepsia focalizada o con epilepsia idiopática generalizada (unos 50 millones de personas en todo el mundo), con claro predominio en aquéllos con epilepsia del lóbulo temporal $(27,28)$.

Su aparición no es inmediata sino que hay una latencia media de 14,4 años tras la aparición de la epilepsia (5). A pesar de su prevalencia, se trata de una entidad nosológica poco estudiada puesto que "se escurre" entre los límites de la Psiquiatría y la Neurología (6).

Su prevalencia entre los enfermos mentales crónicos permanece sin determinar, al menos en la literatura hispánica y anglosajona. Consideramos prudente asumir que un número importante de estos enfermos acaban ingresados en unidades de larga estancia diagnosticados de cuadros psicóticos atípicos y de difícil tratamiento. A favor de ésta opinión está el hecho que la mayoría de los estudios neuropatológicos de pacientes con psicosis intercomicial son antiguos incluyendo únicamente grupos de pacientes con epilepsia del lóbulo temporal en su mayoría ingresados en instituciones psiquiátricas (3).

Los factores de riesgo identificados con ésta patología son: epilepsia de comienzo antes de los 10 años, convulsiones febriles (episodio con una duración superior a los 30 minutos), antecedentes familiares de enfermedad psicótica, aura de predominio autonómico, convulsiones complejas parciales y focalidad en lóbulo temporal $(5,29)$.

Como en los procesos patológicos descritos hasta ahora, la sintomatología es variada, con marcada clínica productiva. En aquellos casos en los que no se puede documentar crisis convulsivas, la sintomatología psiquiátrica: alucinaciones intercomiciales, ilusiones sensitivas y cognitivas, y otras manifestaciones psicopatológicas (ansiedad y depresión); lógicamente motivan la valoración psiquiátrica de una psicosis atípica y son los factores determinantes de la duración del tratamiento psiquiátrico (30).

Aunque no se sabe con certeza, qué mecanismo induce la aparición de las psicosis intercomiciales, sabemos que son más frecuentes en individuos con focos epileptógenicos temporales mediobasales con descargas frecuentes $(3,31)$. 
NOTAS CLÍNICAS

Se barajan dos hipótesis. Inicialmente, un grupo de investigadores, postuló que las convulsiones promueven la psicosis al extenderse al área tegmental ventral y así potenciar la transmisión dopaminérgica en el neocórtex y sistema límbico (teoría "kindling" de las psicosis en la epilepsia), aceptando que la evidencia a su favor no era del todo concluyente (32).

Trabajos más recientes, defienden que la actividad eléctrica desordenada y frecuente induce cambios neuronales en la expresión génica y de proteínas, cambios sinápticos, muerte neuronal, neurogénesis y otros factores, que contribuyen a la creación de nuevos circuitos neurales causantes del trastorno psicótico de la epilepsia tipo esquizofrenia (3). Se propone un modelo bifásico (el "two hit model"), en el que son necesarios por un lado, una hiperactividad en el lóbulo temporal medial (amígdala e hipocampo), además de una hipofunción del lóbulo frontal, en especial del área prefrontal. Éste déficit funcional frontal se traduce en una menor inhibición a los procesos neurales originados en el lóbulo temporal medial, con la consiguiente aparición de sintomatología psicótica. La disregulación funcional temporal medial resultante, podría estar asociada con una valoración emocional y generación de respuestas anormales. Consecuentemente, un proceso continuo aberrante de valoración emocional del entorno y el self, puede resultar en sintomatología psiquiátrica. Se postula que las descargas epiléptica límbicas puede afectar al procesamiento emocional causando un "trastorno emocional transitorio" cuya implicación en la etiología de trastornos psiquiátricos requiere más investigación (6).

Esta última teoría, describe un estado de valoración emocional anormal del entorno y el self, similar al que describe el concepto de "asignación anormal de relevancia" en la esquizofrenia, que a su vez ha sido asociada a actividad en el sistema límbico, núcleo estriado y accumbens, considerado como un mecanismo central en la génesis de la psicosis. Según éste concepto, un papel importante de la dopamina es mediar en la "asignación de relevancia" entre los acontecimientos del entorno y la representación interna que el individuo hace de ellos. Postula que un estado hiperdopaminérgico cerebral conlleva a una asignación aberrante de relevancia a la experiencia personal y proceso "mental". Así, las alucinaciones se entienden como la experiencia directa de asignar una relevancia aberrante a representaciones internas, y las ideas delirantes se entienden como el ajuste cognitivo para entender éstas experiencias relevantes para el individuo. Los fármacos antipsicóticos entumecen la relevancia que el individuo asigna a distintas experiencias, resolviendo la sintomatología productiva. Sin embargo, si se interrumpe el tratamiento antipsicótico, se restablece el desequilibrio neuroquímico produciéndose la recaída en la enfermedad (33).

El siguiente paso consiste en identificar posibles alteraciones estructurales responsables de la disfunción de las estructuras del lóbulo temporal medial. Estudios con neuroimagen estructural han detectado agrandamiento del sistema ventri- 
cular, con reducción del lóbulo temporal, aumento bilateral de la amígdala de hasta el $18 \%$ (en contra de la disminución del tamaño que aparece en la esquizofrenia), alteraciones de la sustancia blanca fronto-temporal (a la que se atribuyen los déficits cognitivos que aparecen en éstos pacientes), displasia del lóbulo temporal, disminución cortical en lóbulo temporal izquierdo y reducción de la materia gris en la circunvolución temporal superior, córtex insular, cerebelo, núcleo caudado y lóbulo parietal inferior izquierdo predatando a la aparición de la psicosis $(12,27,31,34)$.

Los estudios de imagen funcional (SPECT) son menos concluyentes. Por un lado, han detectado hiperperfusión amigdalina y en el lóbulo temporal izquierdo durante episodios psicóticos en pacientes con epilepsia del lóbulo temporal; hipoperfusión en la zona superior del lóbulo temporal izquierdo; o por el contrario no han encontrado diferencia alguna con grupos control. Estudios más recientes, describen una "tendencia" hacia un incremento de flujo cerebral regional en el córtex cingular posterior derecho, hallazgo que se comparte con la esquizofrenia, quizás indicando esta zona como responsable, al menos en parte de la sintomatología productiva (35).

Podemos entonces comprobar cómo algunas de estas anomalías son compartidas con la esquizofrenia. En un estudio con neuroimagen funcional (TEP), se ha constatado hiperactividad temporal medial e hipoactividad frontal durante alucinaciones y utilizando RMN funcional y iEEG, actividad eléctrica aberrante en el lóbulo temporal medial en la paranoia (6).

Otros investigadores, estudiando la actividad eléctrica oscilatoria en EEG durante el descanso, constatan una alteración en el lóbulo parietal, al igual que hizo Sundram (31), que no sólo aparece en individuos con PETE sino también en sujetos con esquizofrenia, enfermedad de Alzheimer, autismo y trastorno por déficit de atención e hiperactividad, pudiendo ser el denominador común de varias enfermedades crónicas con sintomatología psicótica (7).

Teniendo en cuenta los hallazgos de diferentes grupos de investigación tanto en las psicosis de la epilepsia como en la esquizofrenia, sería entonces plausible postular un mecanismo neurobiológico común en ambas patologías, responsable de la génesis de la clínica productiva (6).

Si reflexionamos sobre todo lo expuesto hasta ahora, cabe preguntarse entonces ¿por qué todos los sujetos con epilepsia del lóbulo temporal no presentan cuadros psiquiátricos? La respuesta dista, de momento, de tener una respuesta clara. Habida cuenta del papel que juega la amígdala y estructuras temporales anteriores y mediales en la etiología de trastornos psiquiátricos, y en una analogía con el test de Wada, se postula que una disfunción amigdalina podría ser la causa de éstos trastornos, al producir una disfunción en el sistema límbico, siempre y cuando las estructuras temporales mediales contralaterales no sean capaces de contrarrestar éste efecto. (6). 
El tratamiento de las PETE se lleva a cabo con medicación antipsicótica. Sin embargo, puede ser una tarea difícil ya que los fármacos antipsicóticos pueden causar descargas paroxísticas en el EEG, empeorando el cuadro clínico. Otros problemas asociados al tratamiento con antipsicóticos, incluyen la variabilidad individual del umbral convulsivo (lo que hace a algunas personas más sensibles a los efectos de epileptogénicos de la medicación antipsicótica), interacciones farmacocinéticas y la falta relativa de estudios dedicados a el análisis de la eficacia de los fármacos antipsicóticos en el tratamiento de las psicosis de la epilepsia (3).

Psicosis de la epilepsia tipo esquizofrenia (PETE) o esquizofrenia: un reto diagnóstico.

Probablemente éste sea uno de los mayores desafíos para el clínico en nuestros días. Las manifestaciones clínicas de las psicosis de la epilepsia, en ausencia de signos claros de actividad convulsiva o anomalías electroencefalográficas, son muy difíciles de distinguir de un brote psicótico en el contexto de un proceso esquizofrénico. El médico que busca la certeza diagnóstica, debe apoyarse en otros hallazgos que le guíen hasta un diagnóstico acertado, como por ejemplo alteraciones en el neurodesarrollo, anomalías estructurales en el lóbulo temporal medial, antecedentes de convulsiones febriles, determinados rasgos en la personalidad premórbida, un diagnóstico previo de epilepsia (sobre todo con el foco en el lóbulo temporal), sin olvidar el perfil sintomático del cuadro psicótico.

Los antecedentes familiares, factores genéticos y alteraciones en el neurodesarrollo a pesar de jugar un papel muy importante en la génesis de las psicosis en sujetos con epilepsia, participan en la génesis tanto de la epilepsia como de la esquizofrenia (36). Sin embargo, la epilepsia del lóbulo temporal se asocia a un número de alteraciones estructurales en diferentes áreas cerebrales, no todas concordantes con la esquizofrenia (31).

La edad de aparición del grupo de las psicosis de la epilepsia, tanto comiciales, como postcomiciales e intercomiciales, es sensiblemente posterior a la esquizofrenia, sin detectarse diferencias entre géneros (37).

Lejos de ser un criterio diagnóstico de las PETE, algunos investigadores han analizado la personalidad premórbida de éstos pacientes. Los pacientes diagnosticados de epilepsia generalizada idiopática tienden a ser descritos como inestables, inconsiderados, sin preocuparse por sus obligaciones, fácilmente caen en tentación desviándose de su objetivo, se distraen con facilidad, no tienen en cuenta las posibles consecuencias de sus acciones, pierden control emocional con facilidad, comportándose como el "adolescente perpetuo". Mientras que a las personas con 
un diagnóstico de epilepsia del lóbulo temporal, se les percibe lentos, metódicos, circunstanciales e irritables, pedantes, obstinados, dados a la hipocondría y prácticamente incapaces de cambiar el tema de conversación. Algunos de éstos rasgos se han agrupado en lo que se denomina el síndrome de Geschwind asociado a la epilepsia del lóbulo temporal (38).

Otros aspectos que ayudan a diferenciar las psicosis de la epilepsia de la esquizofrenia crónica incluyen, que en la psicosis de la epilepsia, a pesar de experimentar sintomatología productiva, éstos pacientes permanecen "cálidos y adecuados", mostrando un afecto preservado y durante el curso de la enfermedad no se observa el deterioro de la personalidad propio de la esquizofrenia crónica (39). También destaca que éstos pacientes presentan un mejor funcionamiento pre-mórbido, con una función cognitiva premórbida normal y ausencia de síntomas negativos $(5,40)$; si bien es cierto, que tanto aplanamiento afectivo como retraimiento social se han descrito en una serie de sujetos con epilepsia del lóbulo frontal y psicosis, que los diferenciaba de los pacientes con epilepsia del lóbulo temporal en dicho estudio (4).

\section{Comentario:}

Nos encontramos con un paciente con un diagnóstico de epilepsia de lóbulo temporal y esquizofrenia paranoide que presenta episodios psicóticos de corta duración, que inicialmente responden bien a la administración de benzodiacepinas (lorazepam) estabilizándose su estado mental al añadir clobazam a su tratamiento.

La edad de inicio de la psicosis es más tardía que la que cabría esperar en un esquizofrénico paranoide. El contenido de las ideas delirantes es místico-religioso.

A pesar de una larga evolución, su personalidad no ha sufrido el deterioro que cabría esperar en un esquizofrénico paranoide con la misma evolución. El perfil de su personalidad pre-mórbida es compatible con el que se asocia a la epilepsia del lóbulo temporal.

Además, destaca un buen funcionamiento socio-laboral. Es capaz de terminar los estudios de arquitectura técnica y trabajar como tal, hasta su jubilación temprana por su enfermedad. Además enviuda a una edad joven y, con ayuda de su familia, saca adelante a su hijo con quien convive en la actualidad.

En su estado mental, sobresale un afecto adecuado y una personalidad sin mucho deterioro, a pesar de una edad avanzada y de un deterioro cognitivo crónico, con un componente vascular importante, postulándose una etiología mixta. 
Retrospectivamente, el diagnóstico de episodios psicóticos comiciales parece claro, a pesar de ausencia de anormalidad en el EEG. Sin embargo éste puede ser un proceso difícil de completar, y como bien apuntan Butler et al (6), es muy posible que la mayoría de las psicosis comiciales permanezcan sin ser detectadas o sean mal diagnosticadas.

\section{Conclusión}

No es siempre fácil llegar a un diagnóstico de psicosis de la epilepsia, sin embargo, el clínico debe tenerlo en cuenta en aquellos cuadros psicóticos atípicos, resistentes a tratamiento o con empeoramiento sintomático a la medicación antipsicótica, en personas con un buen ajuste premórbido, y sobre todo en aquéllos previamente diagnosticados de epilepsia del lóbulo temporal. La ausencia de un diagnóstico previo de epilepsia o de anormalidades electroencefalográficas en el EEG convencional, no deben ser óbice para considerar ésta opción diagnóstica.

Las implicaciones que conlleva un diagnóstico acertado pueden transformar la vida de una persona abocada de otro modo, a una institucionalización innecesaria.

BIBLIOGRAFÍA:

(1) García Caballero A, García Lado I, González Hermida J, Recimil MJ, Area R, Manes F, Lamas S, Berrios GE. Validation of the Spanish version of the Addenbrooke's Cognitive Examination in a rural community in Spain. International Journal of Geriatric Psychiatry 2006; 21: 239-45.

(2) Panayiotopoulos C.P. The epilepsies: seizures, syndromes and management. Chipping Norton (UK): Bladon Medical Publishing 2005.

(3) Elliott B, Joyce E, Shorvon S. Delusions, illusions and hallucinations in epilepsy: 2. Complex phenomena and psychosis. Epilepsy Research 2009; 85: 172-86.

(4) Adachi N, Onuma T, Nishiwaki S, Murauchi S, Akanuma N, Ishida S, Takei N. Inter-ictal and post-ictal psychoses in frontal lobe epilepsy: A restrospective comparison with psychoses in temporal lobe epilepsy. Seizure 2000; 9: 328-35.

(5) Adachi N, Akanuma N, Ito M, Kato M, Hara T, Oana Y, Matsuura M, Okubo Y, Onuma T. Epileptic, organic and genetic vulnerabilities for timing of the development of interictal psychosis. British Journal of Psychiatry 2010; 196: 212-16.

(6) Butler T, Weisholtz D, Isenberg N, Harding E, Epstein J, Stern E, Silbersweig D. Neuroimaging of frontal-limbic dysfunction in schizophrenia and epilepsy-related psychosis: Toward a convergent neurobiology. Epilepsy \& Behavior 2012; 23: 113-22. 
(7) Canuet L, Ishii R, Pascual-Marqui RD, Iwase M, Kurimoto R, Aoki Y, Ikeda S, Takahashi H, Nakahachi T, Taakeda M. Resting-state EEG source localization and functional connectivity in schizophrenia-like psychosis of epilepsy. PLoS ONE 2011; 6(11): e27863. Doi:10.1371/journal. pone. 0027863 .

(8) Adachi N, Onuma T, Kato M, Ito M, Akanuma N, Hara T, Oana Y, Okubo Y., Matsuura, M. Analogy between psychosis antedating epilepsy and epilepsy antedating psychosis. Epilesia 2011; 52 (7): 1239-44.

(9) Qin P, Xu H, Laursen TM, Vestergaard M, Mortensen PB. Risk for schizophrenia and schizophrenia-like psychosis among patients with epilepsy: population based cohort study. British Medical Journal 2005; doi:10.1136/bmj.38488.462037.8F (published 17 June 2005).

(10) de Araújo Filho GM, Macedo da Silva J, Mazetto L, Marchetti R L, Yacubian EMT. Psychoses of epilepsy: A study comparing the clinical features of patients with focal versus generalized epilepsies. Epilepsy \& Behavior 2011; 20: 655-58.

(11) Briellmann RS, Kalnins RM, Hopwood MJ, Ward C, Berkovic SF, Jackson GD. TLE patients with post-ictal psychosis: Mesial dysplasia and anterior hippocampal preservation. Neurology 2000; 55: 1027-30.

(12) Terbatz van Elst L, Baeumer D, Lemieux L, Woermann FG, Koepp M, Krishnamoorthy S, Thompson PJ, Ebert D, Trimble MR. Amygdala pathology in psychosis of epilepsy. A magnetic resonance imaging study in patients with temporal lobe epilepsy. Brain 2002; 125: 140-9.

(13) Aroniadou-Anderjaska V, Fritsch B, Qashu F, Braga MFM. Pathology and pathophysiology of the amygdala in epileptogenesis and epilepsy. Epilepsy Research 2008; 78: 102-16.

(14) Gastaut H, Roger J, Roger A. Sur la signification de certaines fugues épileptiques. A propso d'une observation électroclinique d'état de mal temporal. Revue Neurologique 1956; 94: 298-301.

(15) Kanner AM. Psychosis of epilepsy: a neurologist's perspective. Epilepsy \& Behavior 2000; 1: 219-27.

(16) Landolt H. Serial electroencephalographic investigations during psychotic episodes in epileptic patients and during schizophrenic attacks. In: Lorentz de Haas, A.M. (editor). Lectures on Epilepsy. Amsterdam: Elsevier 1958.

(17) Akanuma N, Kanemoto K, Adachi N, Kawasaki J, Ito M, Onuma T. Prolonged post-ictal psychosis with forced normalization (Landolt) in temporal lobe epilepsy. Epilepsy \& Behaviour 2005; 6: 456-59.

(18) Jackson JH. On temporary mental disorders after epileptic paroxysm. West Riding Lunatic Assylum Medical Reports 1875; 5: 105-129.

(19) Kanemoto K, Kawasaki J, Kawai J. Postictal psychosis: a comparison with acute interictal and chronic psychoses. Epilepsia 1996; 37: 551-56.

(20) Logsdail SJ, Toone BK. Post-ictal psychoses: a clinical and phenomenological description. British Journal of Psychiatry 1988; 152: 246-52.

(21) Alper K, Kuzniecky R, Carlson C, Barr WB, Vorkas CK, Patel JG, Carrelli AL, Starner K, Flom PL, Devinsky O. Postictal psychosis in partial epilepsy: A case-control study. Annals of Neurology 2008; 63: 602-10.

(22) Falip M, Carreño M, Donaire A, Maestro I, Pintor L, Bargalló N, Boget T, Raspall A, Rumiá J, Setoaín J. Postictal psychosis: A restrospective study in patients with refractory temporal lobe epilepsy. Seizure 2009; 18: 145-49. 
(23) Chang Y-T, Chen P-C, Tsai I-J, Sung F-C, Chin Z-N, Kuo H-T, Tsai C-H, Chou I-C. Bidirectional relation between schizophrenia and epilepsy: A population-based retrospective cohort study. Epilepsia 2011; 52(11): 2036-42.

(24) Kanner AM, Barry JJ. Controversy in epilepsy and behavior: is the psychopathology of epilepsy different from that of non-epileptic patients? Epilepsy \& Behavior 2001; 2: 170-86.

(25) Farkas M, Baran B, Karpati R, Rajna P. Utility of electroshock therapy in epilepsy-associated psychosis. Ideggyogy Sz. 2002; 55(11-12): 400-5.

(26) Sachdev P. Schizophrenia-like psychosis and epilepsy: The status of the association. American Journal of Psychiatry 1998; 155: 325-36.

(27) Flügel D, Cercignani M, Symms MR, Koepp MJ, Foong J. A magnetization transfer imaging study in patients with temporal lobe epilepsy and interictal psychosis. Biological Psychiatry 2006; 59: 560-67.

(28) Roberts GW, Done DJ, Bruton C, Crow TJ. A “mock up" of schizophrenia: Temporal lobe epilepsy and schizophrenia-like psychosis. Biological Psychiatry 1990; 28: 127-43.

(29) Kanemoto K, Tsuji T, Kawasaki J. Rexamination of interictal psychoses based on DSM IV psychosis classification an international epilepsy classification. Epilepsia 2001; 42(1): 98-103.

(30) Silberman EK, Sussman N, Skillings G, Callanan M. Aura phenomena and psychopathology: a pilot investigation. Epilepsia 1994; 35(4): 778-84.

(31) Sundram F, Cannon M, Doherty CP, Baker GJ, Fitzsimons M, Delanty N, Cotter D. Neuroanatomical correlates of psychosis in temporal lobe epilepsy: voxel-based morphometry study. British Journal of Psychiatry 2010; 197: 482-92.

(32) Smith PF, Darlington CL. The development o psychosis in epilepsy: a re-examination of the kindling hypothesis. Behavioural Brain Research 1996; 75: 59-66.

(33) Kapur S. Psychosis as a state of aberrant salience: a framework linking biology, phenomenology and pharmacology in schizophrenia. American Journal of Psychiatry 2003; 160: 13-23.

(34) Takahashi T, Wood SJ, Yung AR, Walterfang M, Phillips LJ, Soulsby B, Kawasaki Y, McGorry PD, Suzuki M, Velakoulis D, Pantelis C. Superior temporal gyrus volume in antipsychotic-naïve people at risk of psychosis. British Journal of Psychiatry 2010; 196: 206-11.

(35) Guarnieri R, Wichert- Ana L, Hallak JEC, Velasco TR, Walz R, Kato M, Alexandre Jr V, Terra-Bustamante VC, Bianchin MM, Zuardi AW, Deakin JFW, Sakamoto AC. Interictal SPECT in patients with mesial temporal lobe epilepsy and psychosis: a case control study. Psychiatry Research: Neuroimaging 2005; 138: 75-84.

(36) Wotton CJ, Goldacre MJ. Coexistence of schizophrenia and epilepsy: Record-linkage studies. Epilepsia 2012; 53(4): 71-4.

(37) Adachi N, Hara T, Oana Y, Matsuura M, Okubo Y, Akanuma N, Ito M, Kato M, Onuma T. Difference in age of onset of psychosis between epilepsy and schizophrenia. Epilepsy Research 2008; 78 : 201-6.

(38) Janz D. The psychiatry of idiopathic generalized epilepsy. In: Trimble M, \& Schmidt B. (editors). The neuropsychiatry of epilepsy. Cambridge University Press: Cambridge; 2002.

(39) Trimble MR. The psychoses of epilepsy. New York : Raven Press; 1991.

(40) Matsuura M, Trimble MR. Psychosis in epilepsy: a review of Japanese studies. Epilepsy \& Behavior 2000; 1: 315-26. 